\title{
Diferenças entre Chenopodium quinoa e A Planta Daninha Chenopodium album
}

\author{
Differences Between Chenopodium quinoa and the Weed Chenopodium album
}

\author{
SPEHAR, C.R. ${ }^{2}$, SANTOS, R.L.B. ${ }^{3}$ e NASSER, L.C.B. ${ }^{3}$
}

\begin{abstract}
RESUMO - A experimentação pioneira com Chenopodium quinoa Willd tem demonstrado sua adaptabilidade à produção de grãos no cerrado. Seus frutos, do tipo aquênio, são cilíndricos, achatados e germinam rapidamente na presença de umidade, após a maturação fisiológica. $\mathrm{Na}$ fase inicial do seu desenvolvimento, a quinoa pode ser confundida com a planta daninha Chenopodium album, conhecida no Brasil como ançarinha-branca. As diferenças básicas entre as duas espécies se tornam mais visiveis após o florescimento: ramificação profusa, com rácemos axilares e terminais em C. album, em contraste com C. quinoa, na qual as panículas são terminais, à semelhança do sorgo; o pericarpo é claro e contrasta com o preto em C. album. A quinoa BRS Piabiru, primeiro cultivar para o Brasil, apresenta plantas com $190 \mathrm{~cm}$, nas quais a panícula ocupa $45 \mathrm{~cm}$; maturação fisiológica aos 145 dias; resistência

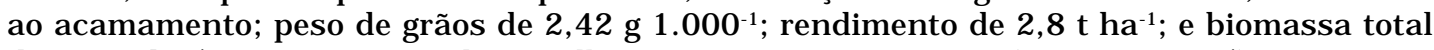
de $6,6 \mathrm{t} \mathrm{ha}^{-1}$. As sementes de $C$. album são muito pequenas $\left(0,52 \mathrm{~g} 1.000^{-1}\right)$, germinam gradativamente e permanecem no solo por muitos anos, infestando os cultivos. As diferenças no número de cromossomos, impedindo a polinização cruzada entre as duas espécies e as morfológicas, detectadas na experimentação, mostram que estas são distinguiveis e asseguram que a quinoa apresenta características de adaptação ao cultivo comercial, contrapondo-se às características de invasora em C. album.
\end{abstract}

Palavras-chave: quinoa, ançarinha-branca, morfologia.

\begin{abstract}
Pioneer experimentation with Chenopodium quinoa Willd has shown its adaptability to grain production in the Brazilian savannah. Its fruits (achene type) are cylindrical, flat and germinate quickly in the presence of moisture, after reaching physiological maturity. At its early phase of development, quinoa can be confounded with the weed Chenopodium album, known in Brazil as 'ançarinha- branca'. Their basic differences become more visible after flowering: profuse branching, with axilary and terminal racemes in $\mathbf{C}$. album, contrasting with C. quinoa, whose panicles are terminal and similar to sorghum; the pericarp has a light colour, contrasting with black in C. album. Quinoa BRS Piabiru, the first quinoa cultivar brought to Brazil, presents $190 \mathrm{~cm}$ height, of which $45 \mathrm{~cm}$ is occupied by the panicle; physiological maturity

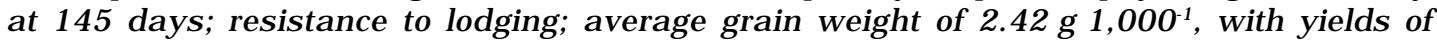
$2.8 \mathrm{tha}^{-1}$ (grains) and $6.6 \mathrm{tha}^{-1}$ (total biomass). The $\mathrm{C}$. album seeds are very small $\left(0.52 \mathrm{~g} \mathrm{1}^{1, O O O^{-1}}\right)$, germinate gradually and remain in soil for many years infesting crops. The differences in number of chromosome leading to impediment to natural crosses, and morphological differences detected during experimentation, show that the two species are distinguishable and that quinoa displays adaptability characteristics for commercial cultivation, contrasting with C. album, which displays typical weed behaviour.
\end{abstract}

Key words: quinoa, 'ançarinha-branca' weed, and morphology.

Recebido para publicação em 16.1.2003 e na forma revisada em 12.12.2003.

2 Eng.-Agr., Ph.D., Pesquisador, Líder de Projeto, EMBRAPA-Centro de Pesquisa Agropecuária dos Cerrados, Caixa Postal 08223, 73301-970 Planaltina-DF; ${ }^{3}$ Eng.-Agr., M.S., Esplanada dos Ministérios - Bloco 'D' - Edifício Anexo ‘A', Térreo, sala 7, 70043-900 Brasília-DF.

Planta Daninha, Viçosa-MG, v.21, n.3, p.487-491, 2003 


\section{INTRODUÇÃO}

A partir de 1990, a Embrapa, apoiada pelo $\mathrm{CNPq}$, tem realizado trabalho pioneiro com Chenopodium quinoa Willd. para adaptá-la ao cultivo no Brasil (Spehar \& Souza, 1993). A quinoa, ou quínua, é originária dos Andes, onde tem sido cultivada há milênios (Tapia, 1997).

A qualidade da proteína, comparável à caseína do leite, torna a quinoa um cultivo potencial. Por ser mais equilibrada, pode complementar a alimentação de humanos, aves e suínos com vantagem sobre o milho e a soja, quando utilizados isoladamente (Spehar, 2002). Apresenta demanda crescente no mundo, principalmente como alternativa protéica de qualidade com baixo colesterol e por pacientes celíacos - pessoas que apresentam alergia ao glúten (Spehar et al., 1997).

Na perspectiva de ser produzida em larga escala no Brasil, como alternativa de sucessão (safrinha) no sistema plantio direto, o grão poderá ser utilizado pelas indústrias de alimentos e rações (Spehar, 2002). A planta inteira, como forragem na alimentação animal, pode integrar o sistema lavoura-pecuária.

Pela semelhança, no início da fase vegetativa, a quinoa pode confundir-se com a planta daninha Chenopodium album, conhecida no Brasil como ançarinha-branca ou erva-formigueira-branca, o que pode causar problemas no cultivo comercial. Esta ocorre como planta invasora no Sul e, mais recentemente, foi identificada no cerrado (Nascimento, 2000). Acredita-se que tenha sido introduzida via sementes de trigo, nabo forrageiro e feijão, provenientes do Sul ou Sudeste.

A espécie Chenopodium quinoa apresenta ciclo entre 80 e 150 dias, dependendo da variedade, nas condições do Brasil Central. Após 30 dias da semeadura, o crescimento é rápido e as variedades mais tardias atingem cerca de $2,0 \mathrm{~m}$ em semeaduras de safrinha (outono), com suprimento de $300 \mathrm{~mm}$ de umidade (Spehar \& Santos, 2002). A ocorrência e a deposição de oxalato de cálcio nas folhas lhe possibilitam reter umidade, característica desejável na tolerância à seca. Os frutos, do tipo aquênio, são achatados e sem dormência (Tapia, 1997). Por constituírem o material colhido, estes frutos denominam-se sementes.
Quando amadurecem, apresentam rápida germinação na presença de umidade.

BRS Piabiru, o primeiro cultivar de quinoa recomendado em nosso país (Spehar \& Santos, 2002), é descrito pelas seguintes características botânicas: hipocótilo com coloração variável entre verde e rósea; folhas polimórficas, com número de dentes entre 12 e 20 e deposição de oxalato de cálcio, em forma de grânulos, perceptiveis ao toque dos dedos; caule ereto, verde ou verde com estrias; inflorescência diferenciada e terminal, amarantiforme e laxa, amarela, quando a planta atinge a maturação fisiológica; grãos (frutos do tipo aquênio) de forma cilíndrica e achatada, com pericarpo branco; e perigônio (estrutura que envolve o fruto) verde, com ausência de saponina (Spehar \& Santos, 2002). Essa substância, um glicosídeo de sabor amargo, encontra-se presente nas primeiras variedades selecionadas no Brasil (Spehar \& Souza, 1993).

A estatura média (dados médios de campo) em BRS Piabiru é de $190 \mathrm{~cm}$, da qual a inflorescência ocupa $45 \mathrm{~cm}$; diferenciação floral aos 30 dias após a emergência, com a antese aos 45 dias; período entre a emergência e a maturação fisiológica de 145 dias; resistência ao acamamento. Os grãos (frutos), com umidade

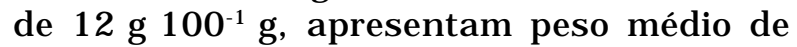

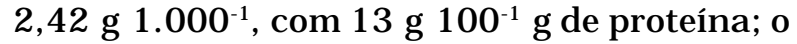
rendimento médio é de $2,8 \mathrm{t} \mathrm{ha}^{-1}$ (grãos) e 6,6 t ha-1 (biomassa total) (Spehar \& Santos, 2002).

As plantas de ançarinha-branca são menores, ramificadas, e suas sementes, também fruto do tipo aquênio, são escuras, dormentes. Guando amadurecem, desprendem-se das plantas e caem ao solo, onde permanecem; germinam gradativamente e infestam as áreas agrícolas.

Este trabalho objetivou estabelecer parâmetros de diferenciação morfológica entre C. quinoa, domesticada, e C. album, planta daninha.

\section{MATERIAL E MÉTODOS}

Um experimento, com delineamento inteiramente casualizado e quatro repetições, foi conduzido em vasos, em casa de vegetação. O substrato foi composto por uma parte de 
matéria orgânica (composto) e duas de solo. Essa mistura foi adubada para atender a demanda das plantas até que atingissem a maturação fisiológica. Três genótipos de quinoa - Piabiru 54, Piabiru 44 (seleções de 'BRS Piabiru') e GunB - e um de C. album, procedente da Universidade de Brasília, constituíram os tratamentos. Quinze sementes por tratamento foram semeadas; o desbaste deu-se quando as plantas apresentavam a primeira folha verdadeira totalmente expandida, deixando-se três plantas por vaso. A umidade foi mantida mediante o uso de bandejas sob os vasos, as quais receberam a mesma quantidade de água, suficiente para suprir a necessidade das plantas. Os turnos de rega se repetiram a cada dois dias.

Durante a condução, avaliaram-se as seguintes características: comprimento, largura (cm), número de dentes e presença de oxalato de cálcio ( 1 - menos denso; 3 - mais denso, pelo aspecto visual) da quarta folha totalmente expandida, após as cotiledonares (base) e abaixo da base da panícula (ápice); comprimento do pecíolo das respectivas folhas; ramificação do caule ( 1 - pouco ramificado; 5 - muito ramificado); tipo e comprimento da inflorescência; ocorrência da antese (dias após a emergência); e fruto - tamanho, cor, quantidade e germinação. Os dados relativos ao número de sementes/planta e o peso de 1.000 sementes, para cada tratamento, foram analisados estatisticamente, sendo as médias comparadas pelo teste de Tukey.

\section{RESULTADOS E DISCUSSÃO}

Os dados experimentais mostram que as folhas, em C. quinoa e C. album, são maiores na base e menores no ápice (Tabela 1), porém diferem consideravelmente no tamanho da lâmina - maior em C. album. O número de dentes é maior em C. album, com menor densidade de grânulos de oxalato de cálcio. As diferenças no tamanho de pecíolo não se mostraram tão evidentes quanto na folha. O caule é pouco ramificado em $C$. quinoa, oposto ao que ocorre com C. album. O número de dias da emergência à antese é variável e depende do ciclo da variedade. Assim, há variedades mais tardias e mais precoces em relação a C. album. As panículas, que podem variar de laxas (abertas) a compactas, são maiores em C. quinoa; em
C. album apresentam-se como racemosas (apicais e axilares).

A quantidade de sementes/planta não foi fator de deferenciação, porém a diferença no seu tamanho foi marcante. As sementes em C. album são pretas, pequenas e pesam $0,52 \mathrm{~g} 1.000^{-1}$, em contraste com tonalidades claras em C. quinoa, cujo peso se mostrou superior a 2,2 g $1.000^{-1}$ (Tabela 1 ). As primeiras germinam gradativamente - apenas a quarta parte - 30 dias após a maturação fisiológica. Permanecem no solo por longo período e podem infestar as áreas agrícolas, enquanto as de quinoa não apresentam dormência e, ao contrário, deterioram ou germinam na panícula, em condição de alta umidade, após a maturação (Spehar \& Souza, 1993).

Os dados evidenciam que as espécies C. quinoa e C. album apresentam características morfológicas diferenciais. A comparação da folha, da planta, da inflorescência e da semente (Figura 1) ilustra as diferenças mencionadas e serve de orientação para distinguir as duas espécies.

A baixa taxa de germinação das sementes em C. album e a morfologia - folha com maior número de dentes, planta mais ramificada, racemos difusos, com menor tamanho da inflorescência terminal e sementes dormentes - são fatores de diferenciação em relação a $C$. quinoa. Esta apresenta-se com maturação coincidente, uniforme, panículas concentradas no topo (favorável à colheita) e sementes claras de germinação plena após atingirem a maturação fisiológica.

As diferenças entre C. quinoa e C. album ocorrem ainda em nível citogenético, com o número de cromossomos de $2 n=36$ e $2 n=54$, respectivamente, ambas com comportamento típico de aloplóides (Cole, 1962). A diferença na ploidia, entretanto, impede que haja cruzamentos naturais, altamente indesejáveis no cultivo comercial (Simonds, 1965).

As características morfológicas e genéticas entre C. quinoa e C. album são suficientes para separar as duas espécies: a primeira, planta domesticada, com conformação adequada à colheita, sementes grandes e sem dormência, difere da ançarinha-branca, planta daninha típica, ramificada, com sementes menores, pretas e dormentes.

Planta Daninha, Viçosa-MG, v.21, n.3, p.487-491, 2003 
Tabela 1 - Características fenotípicas em Chenopodium quinoa e C. album relativas a folha, inflorescência e fruto (semente). Embrapa Cerrados, Planaltina-DF, 2002

\begin{tabular}{|c|c|c|c|c|c|c|c|c|}
\hline \multirow{4}{*}{ Caráter } & \multicolumn{8}{|c|}{ Genótipo } \\
\hline & \multicolumn{6}{|c|}{ Chenopodium quinoa } & \multirow{2}{*}{\multicolumn{2}{|c|}{ C. album }} \\
\hline & \multicolumn{2}{|c|}{ Piabiru 54} & \multicolumn{2}{|c|}{ Piabiru 44} & \multicolumn{2}{|c|}{ Q UnB } & & \\
\hline & Base & Ápice & Base & Ápice & Base & Ápice & Base & Ápice \\
\hline \multicolumn{9}{|c|}{ Folha } \\
\hline Comprimento $(\mathrm{cm})$ & 60 & 35 & 67 & 40 & 67 & 42 & 90 & 50 \\
\hline Largura (cm) & 65 & 21 & 72 & 25 & 55 & 12 & 75 & 25 \\
\hline Pecíolo (cm) & 70 & 36 & 70 & 38 & 53 & 28 & 65 & 35 \\
\hline Dentes/folha & 18 & - & 24 & - & 12 & - & 44 & - \\
\hline Oxalato de $\mathrm{Ca}^{*}$ & 2 & 3 & 2 & 3 & 2 & 3 & 1 & 2 \\
\hline \multicolumn{9}{|c|}{$* 1=$ menos denso; $3=$ mais denso } \\
\hline \multicolumn{9}{|c|}{ Planta/Inflorescência } \\
\hline Ramificação** & \multirow{4}{*}{\multicolumn{2}{|c|}{$\begin{array}{c}2 \\
62 \\
\text { Laxa } \\
35\end{array}$}} & \multicolumn{2}{|c|}{2} & \multicolumn{2}{|c|}{2} & \multicolumn{2}{|r|}{4} \\
\hline Antese $* * *$ & & & \multicolumn{2}{|c|}{54} & \multicolumn{2}{|c|}{44} & \multicolumn{2}{|r|}{54} \\
\hline Panícula & & & \multicolumn{2}{|c|}{ Laxa } & \multicolumn{2}{|c|}{ Compacta } & \multicolumn{2}{|r|}{ Racemosa } \\
\hline Comprimento $(\mathrm{cm})$ & & & \multicolumn{2}{|c|}{30} & \multicolumn{2}{|c|}{30} & \multicolumn{2}{|r|}{20} \\
\hline \multicolumn{9}{|c|}{$\begin{array}{l}* * 1 \text { = pouco ramificado; muito ramificado. } \\
* * * \text { número de dias após a emergência. }\end{array}$} \\
\hline \multicolumn{9}{|c|}{ Fruto } \\
\hline Peso $\left(\mathrm{g} 1.000^{-1}\right)^{)^{1 /}}$ & \multirow{4}{*}{\multicolumn{2}{|c|}{$\begin{array}{c}2,23 \mathrm{a} \\
\text { Branca } \\
1.042 \mathrm{~b} \\
99\end{array}$}} & \multirow{4}{*}{\multicolumn{2}{|c|}{$\begin{array}{c}2,41^{\mathrm{a}} \\
\text { Branca } \\
1.297^{\mathrm{a}} \\
100\end{array}$}} & \multirow{4}{*}{\multicolumn{2}{|c|}{$\begin{array}{c}2,21^{\mathrm{a}} \\
\text { Amarela } \\
985 \mathrm{~b}\end{array}$}} & \multirow{4}{*}{\multicolumn{2}{|c|}{$\begin{array}{c}0,52 b \\
\text { Preta } \\
1.031 b\end{array}$}} \\
\hline Cor & & & & & & & & \\
\hline Número/Planta ${ }^{1 /}$ & & & & & & & & \\
\hline Germinação**** & & & & & & & & \\
\hline
\end{tabular}
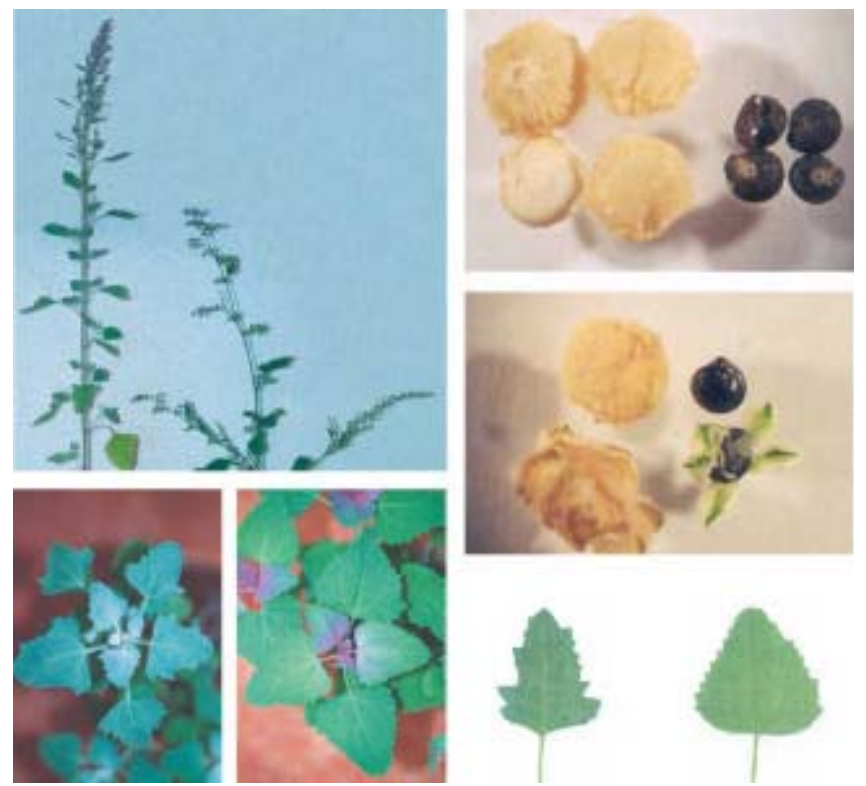

Figura 1 - Morfologia de Chenopodium quinoa (esquerda) e C. album (direita): folha, planta, inflorescência e semente (com e sem perigônio - estrutura oriunda do cálice, que envolve o fruto). 


\section{LITERATURA CITADA}

COLE, M. J. Interspecific relationship relations and intraspecific variation of Chenopodium album L. in Britain. II. The Chromosome number of $C$. album and other species. Watsonia, v. 5, p. 117-122. 1962.

NASCIMENTO, E. P. Chenopodium album, um problema no cerrado. R. Bras. Planta Daninha, v. 1, n. 1, p. 1, 2000.

SIMONDS, N. W. The grain chenopods of the tropical American highlands. Econ. Bot., v. 19, p. 223-235, 1965.

SPEHAR, C. R. Production systems in the savannas of Brazil: key factors to sustainability. In: LAL, R. (Ed.) Soil quality and agricultural sustainability. Chelsea: Ann Arbor Press, 1998. p. 301-318.

SPEHAR, C. R. Utilização da quinoa como alternativa para diversificar alimentos. In: SIMPÓSIO SOBRE INGREDIENTES NA ALIMENTAÇÃO ANIMAL. Uberlândia, MG: Colégio Brasileiro de Nutrição Animal/ UFU. 2002. p. 49-58.
SPEHAR, C. R.; CABEZAS, W. A. R. L. Introdução e seleção de espécies para a diversificação do sistema produtivo nos cerrados. In: CABEZAS, W. A. R. L.; FREITAS, P. L. (Eds.) Plantio direto na integração lavoura pecuária. Uberlândia: UFU, 2000. p. 179-188.

SPEHAR, C. R.; SANTOS, R. L. B. Quinoa (Chenopodium quinoa Willd) BRS Piabiru: alternativa para diversificar os sistemas de produção de grãos. Pesq. Agropec. Bras., v. 37 , n. 6 , p. $889-893,2002$.

SPEHAR, C. R.; SANTOS, R. L. B.; SOUZA, P. I. M. Novas espécies de plantas de cobertura para o plantio direto. In: SEMINÁRIO INTERNACIONAL DO SISTEMA

PLANTIO DIRETO, 2., 1997, Passo Fundo. Anais... Passo Fundo: EMBRAPA-Trigo, 1997. p. 169-172.

SPEHAR, C. R.; SOUZA, P. I. M. Adaptação da Quinoa (Chenopodium quinoa Willd.) ao cultivo nos cerrados do Planalto Central: resultados preliminares. Pesq. Agropec. Bras., v. 28, n. 5, p. 635-639, 1993.

TAPIA, M. Cultivos andinos subexplotados y su aporte a la alimentación. Santiago, Chile: Oficina Regional de la FAO para la América Latina y Caribe, 1997. 217 p. 